\title{
Correlation of Left Ventricular Ejection Fraction and Spatial QRS-T Angle In Old Myocardial Infarct Patient
}

\author{
Giky Karwiky, Erwan Martanto, Ferdy Sanjaya, Chaerul Achmad
}

Department of Cardiology and Vascular Medicine, Faculty of Medicine, Universitas PadjadjaranDr. Hasan Sadikin Hospital, Bandung

\section{Correspondence:}

Giky Karwiky

Staff of Electrophysiology Division, Department of Cardiology and Vascular Medicine, Hasan Sadikin General Hospital

Email: giky.karwiky@unpad.ac.id

\begin{abstract}
Background: Half of patients with coronary artery disease (CAD) died from sudden cardiac death (SCD) with Left Ventricular Ejection Fraction (LVEF) as a predictor. Spatial QRS-T angle (the angle between the QRS and T vectors) is a strong independent predictor of cardiovascular death. The aim of this study was to correlate spatial QRS-T (sQRST) with LVEF in patients with old myocardial infarction (OMI).

Methods: This is a cross-sectional study in patients with OMI that have not undergone revascularization and have achieved medical therapy. OMI was defined based on universal definition of myocardial infarction electrocardiography (ECG) criteria. I2-lead ECG and echocardiography were done simultaneously. sQRST angle was measured by Kors visual transform applications. Statistical analysis was performed using Pearson correlation and multivariate analysis with linear regression.

Results: 46 patients meet the inclusion criteria. Baseline characteristics: mean age $58 \pm 8$ years, $89 \%$ male, mean sQRST was $108.72 \pm 43^{\circ}$ with mean LVEF $39.39 \pm 10 \%$. The sQRST angle and LVEF was strong negative correlation $(r=-0.66, p<0.0 \mathrm{I})$ after adjusted with left ventricular mass index (LVMI) correlation between sQRST angle and LVEF decreasing $(r=-0.57, p<0.0 \mathrm{I})$. The SQRST angle and LVEF of patients with OMI is negative correlation.

Conclusion: The sQRST angle and LVEF of patients with OMI had negative correlation. SQRST angle may be an easier index for assessing cardiac dysfunction in patients with OMI.
\end{abstract}

(Indonesian J Cardiol. 202 I;42:4 I-45)

Keywords: Left ventricular ejection fraction, Old myocardial infarction, Spatial QRS-T angle 


\section{Introdution}

$\mathrm{T}$

hesQRST angle measures the difference in the angle of the QRS wave vector and the $T$ wave vector in 3 dimensions. Wide QRS-T angle is associated with increased cardiovascular events, high mortality and SCD mainly due to increased ventricular arrhythmias. ${ }^{1}$ Existing studies showed that the sQRST angle can predict cardiovascular events, SCD, or overall mortality better than other conventional ECG markers such as ST segment depression, T wave inversion, left ventricular hypertrophy, increased QT interval, and $\mathrm{T}$ wave dispersion. ${ }^{1-3}$ Wide $s \mathrm{QRST}$ angle $\left(>100^{\circ}\right)$ is a strong predictor (7-fold increased risk) of developing ventricular arrhythmias. The gold standard tool for measuring the sQRST angle was to use vectorcardiography proposed by Frank using orthogonal leads X, Y, and Z. ${ }^{4}$ This lead system was impractical so it is rarely used in daily clinical applications. Researchers have attempted to calculate sQRST angle by reconstructing Frank's vectorcardiogram with the commonly used 12-leads ECG. Based on existing studies regarding the comparison between various methods of measuring the spatial angle of QRS-T waves using a 12-leads ECG, the Kors matrix calculation is considered the best because it shown the closest result to Frank's vectorcardiogram measurement. ${ }^{5-6}$

Myocardial necrosis will lead to a prolonged action potential of the heart, and heterogeneity of repolarization. Changes in the cellular level in the area of necrosis cause disruption of the electrical flow of the heart to the surrounding area. This change $\rightarrow$ in action potential morphology will cause changes in the entire QRS-T complex which are recorded as spatial changes in the QRS-T wave. ${ }^{7}$ Necrotic area will cause an inertia region towards cardiac electrical activity, causing distortion of normal cardiac excitation and inhomogeneity of myocardial repolarization. This will cause the sQRST angle to widen. ${ }^{1}$

Research conducted by $\mathrm{Li}$ et al. ${ }^{8}$ using the frontal QRS-T angle had an inverse correlation with ejection fraction. The lower the ejection fraction, the wider the frontal QRS-T angle with an angle of $>90^{\circ}$ has the optimal sensitivity and specificity $(76 \%$ sensitivity and $74 \%$ specificity) for diagnosis of ventricular dysfunction. Research conducted by Brown et al. stated that sQRST angle was more accurate than the frontal QRS-T angle in the case of cardiac diseases. ${ }^{9}$ This study is different from previous studies because it tries to correlate the spatial QRS $t$ angle as a more accurate marker of sudden cardiac death in old myocardial infarction patients who have not been revascularized but have received drug therapy.

\section{Methods}

This is a cross-sectional study conducted at Hasan Sadikin Hospital, Bandung from October to December 2015. We recruited subjects with OMI who were on regular follow up at cardiology outpatient clinic and inpatient ward receiving medical therapy without undergoing revascularization procedure. We excluded patients with acute coronary syndrome, stable angina pectoris, diabetes mellitus, end-stage renal disease, acute stroke, arrhythmia, and left or right complete bundle branch block. The study protocol was reviewed and approved by Research Ethics Committee, Faculty of Medicine, Padjadjaran University, Bandung.

Patients were given informed consent, had clinical history taken, physical exam and additional examination. Patients who meet the inclusion criteria as OMI will be included in the study and treated according to the applicable procedure. Supporting examinations performed on the patient is an ECG, echocardiography, and blood sample (fasting blood sugar and blood sugar 2 hours post prandial). The Simpson method was used to measure left ventricular ejection fraction (LVEF) by echocardiography machine (GE Vivid 7). Identical electrocardiographs (Philips Page Writer TC50) were used and standard 12-lead ECGs were recorded for all subjects using strictly standardized procedures. Calculation of the QRS-T spatial angle with an ECG, usually done with a system called the spatial peak angle of the QRS-T wave which only requires the peak voltage value of the QRS wave and the $T$ wave. This peak voltage value can be seen easily on an ordinary 12 lead ECG. These numbers are then entered into the desired QRS-T spatial angle transformation formula. We used Kors regression matrix because closely related to Frank leads as gold standard. 5

Statistical analysis was carried out in accordance with the proposed objectives and hypotheses, starting with a descriptive description, data normality test, then a correlation test analysis was carried out between the 
LVEF and the spatial angle of the QRS-T wave using Pearson correlation analysis if the data was linear or using Spearman correlation if the data is non-linear. Confounding variables that have not been eliminated at the research design stage will be analyzed through multivariate analysis. Statistical calculations were performed using the International Business Machine (IBM) Software Package for Statistical Analysis (SPSS) version 17.

\section{Results}

A total of 53 patients met the inclusion criteria, and 7 patients were excluded with the following reasons: four patients with a diagnosis of stable angina pectoris; two patients with uninterpretable data and one patient with left-branch bundle block. The general characteristics of the study subject can be seen in table 1 below.

In this study, the average age was 58 (8) years. Men $(89 \%)$ is more than women (11\%). Smoking is the most common risk factor $(74 \%)$ followed by hypertension (50\%), dyslipidemia (32\%) and family history (6\%). Majority of patients received antiplatelet agents $(91 \%)$; the rest received warfarin (6\%). As many as $85 \%$ of OMI patients received betablockers, Angiotensin Converting Enzyme (ACE) inhibitors (63\%), Statins (72\%), Angiotensin Receptor Blockers (22\%), Diuretics (54\%), Aldosterone Antagonists (17\%). Anterior OMI was more common than inferior OMI (67\% vs $39 \%)$. Half of OMI patients (50\%) had no known onset of first-time heart attack, while the other half (50\%) had a documented history of previous infarction with a mean onset 5 months after infarction.

Examination of the sQRST angle and LVEF in the study subjects listed in Table 2. The median sQRST angle was $108.72^{\circ}$ and the mean LVEF was $39.39 \%$.

Based on table 2, the analysis to determine the relationship between the spatial angle of the QRS-T wave and the LVEF was carried out using the Pearson correlation test because the data were normally distributed. The results obtained are as shown in table 3 and Figure 1 which are mapped in a scatter plot.

From Figure 1. it can be concluded that there is a strong negative correlation between the sQRST angle and the LVEF with $r=0.66$ and $p<0.05$. This means that the higher the sQRST angle, the lower the LVEF.

sQRST Angle also correlated significantly with left ventricular mass index (LVMI) ( $\mathrm{p}=0.001, \mathrm{r} 0.449)$ with moderate level of correlation. The larger the LVMI, the greater the spatial angle.

After performing the bivariate analysis, all variables met the criteria for multivariate analysis. All linear regression assumptions (i.e., linearity, residual normality, no outliers, independent, constant, and homoscedasticity) were met. After confounding variables (LVMI) was controlled, it was found that the correlation between the sQRST angle and the LVEF was still significant but with moderate correlation $(\mathrm{p}=0.00$ $r=-5.76$ ). This shows that after adjusting the LVMI the correlation with the spatial angle is moderate, showing contribution of LVMI to the correlation between spatial angles and LVEF.

\section{Discussion}

A metanalysis involving 22 studies, comprising 164,171 individuals of a diverse population, concluded that a wide QRS-T wave angle was associated with increased total mortality (RR: 1.40; 95\% CI (confidence interval): 1.32-1.48) and cardiac mortality (RR). : 1.71; 95\% CI: $1.54-1.90) .{ }^{10}$ On the other hand, LVEF is currently accepted as the main prognostic tool in predicting SCD. ${ }^{11}$ Based on the existing literature, this study is the first to correlate the sQRST angle and LVEF.

The results of this study showed a strong negative correlation between the sQRST angle and the LVEF ( $\mathrm{r}$ $=-0.66$ and $p<0.05)$ but after adjusting for LVMI the correlation became moderate $(\mathrm{r}=-0.57$ and $\mathrm{p}<0.05)$ so it can be concluded that the higher the sQRST angle, the lower the LVEF. Zapolski et al. found a strong correlation between the spatial angle of the QRS-T wave and left ventricular contractility calculated using $\mathrm{mFS} / \mathrm{ESS}$ (mid wall fractional shortening/end systolic stress). ${ }^{12}$ Studies in diabetics show a strong correlation between the sQRST angle and Tei index, an indicator of left ventricular contractility performance that combines systolic and diastolic functions. ${ }^{13}$ Yan Hong $\mathrm{Li}$ et al. found a moderate negative correlation between the frontal QRS-T angle and the LVEF $(r=-0.406, p$ $<0.01$ ) in patients with OMI. ${ }^{8}$ The findings in this study corroborate the opinion of Brown et al. that spatial angle is better in detecting structural heart disease than frontal angle.

This study uses the peak calculation of the QRS-T 
vector with the Kors matrix compared to the calculation of the average QRS-T vector because many studies recommend the calculation of the peak vectors. Cortez et al. revealed that the value of the sQRST angle in patients after myocardial infarction is close to examination value of Frank standardization with a strong correlation level (0.83). ${ }^{5}$ The value of the spatial angle of the QRS-T wave after myocardial infarction in this study was higher than that of Cortez et al. The average value of Cortez's spatial angle was $76.4^{\circ} \pm 42$, while in this study the mean value was $108.72^{\circ} \pm 43$. Cortez's study had similar baseline sample characteristics with a mean age of $58.8 \pm 11.3$ years and $72 \%$ male gender. The difference was that the patient in Cortez's study was a recent myocardial infarction but the average onset of attacks that occurred was not mentioned. In this study $50 \%$ patients had an attack onset of 5 months, while the other $50 \%$ had no information regarding onset of previous attacks, yet this indicates a longer mean post-attack time in this study. 5 Along with the continuous remodeling process, the sQRST angle will also get wider. ${ }^{12}$

This study also found a moderate correlation between the sQRST angle and the LVMI ( $\mathrm{p}=0.001, \mathrm{r}$ 0.449 ). This finding is consistent with previous studies that the greater the LVMI, the wider the spatial angle will be. A total of 25 patients (54\%) had severely abnormal LVMI (male $\geq 149 \mathrm{~g} / \mathrm{m} 2$, female $\geq 122 \mathrm{~g}$ / $\mathrm{m} 2) .{ }^{12}$ After controlling confounding variables (LVMI), it was found that correlation between the sQRST angle and LVEF still significant but with moderate correlation $(\mathrm{P}<0.001 \mathrm{r}=-5.76)$. This result shows that after adjusting the LVMI, the correlation with spatial angle become moderate, pointing contribution of LVMI to the correlation between spatial angles and LVEF.

The correlation between the sQRST angle and the LVEF is not perfect, meaning that not all patients with $\mathrm{LVEF} \leq 35 \%$ have high spatial angles. The value of the sQRST angle is divided into normal $\left(0-105^{\circ}\right)$, borderline $\left(105-135^{\circ}\right)$, and abnormal $\left(135-180^{\circ}\right) .3$ In this study, $14 \%$ of patients with LVEF $\leq 35 \%$ had borderline spatial angles, and $7 \%$ of patients had normal spatial angles. In these patient, electrical homogeneity occurs and is thought to have a limited arrhythmia substrate. Borleff et al. concluded that post-myocardial infarction patients with low LVEF attached to an ICD with a normal spatial angle did not develop fatal arrhythmias during 2 years of follow-up and the risk of subsequent arrhythmias was only $2 \%$ compared to those with a wide spatial angle who had 7 folds increased risk of ventricular arrhythmias. 15 On the other hand, 21\% of patients with LVEF $\geq 35 \%$ had abnormal spatial angles. Based on these findings, the spatial angle of the QRS-T wave can identify patients with a low risk of developing arrhythmias so that it can complement the LVEF in stratifying the risk of SCD. Its application in daily clinical practice needs further investigation.

This study has several limitations which include echocardiography and sQRST angle were only performed by one operator so that interobserver variability could not be assessed. This study also cannot rule out left ventricular hypertrophy with the LVMI criteria so that the relationship between the sQRST angle and the LVEF is influenced.

In conclusion, there is a moderate negative correlation between the spatial angle of the QRS-T wave and the LVEF in patients with OMI after multivariate analysis with LVMI. Increase in the sQRST angle can be used as an indicator of a decrease in LVEF in patients with OMI.

\section{Publication Approval}

All authors read and approved the final manuscript.

\section{Conflict of Interest}

None.

\section{Sources of Funding}

This paper received no specific grant from any funding agency, commercial or not-for-profit sectors.

\section{Ethical Clearance}

We proved ethical clearance from our ethical committee along with this submission.

\section{Copyright/Permission for Figures}

We the Authors of this manuscript declare that we have received the proper permission regarding the use of figures/images/illustrations from their original author. 


\section{List of Abbreviations}

ACE: Angiotensin Converting Enzyme

CI: Confidence Interval

CAD: Coronary Artery Disease

ECG: Electrocardiography

IBM: International Business Machine

LVEF: Left Ventricular Ejection Fraction

LVMI: Left Ventricular Mass Index

OMI: Old Myocardial Infarction

RR: Relative Risk

SPSS: Software Package for Statistical Analysis

sQRST: Spatial QRS-T

SCD: Sudden Cardiac Death

\section{References}

1. Voulgari C, Pagoni S, Tesfaye S, Tentolouris N. The Spatial QRS-T angle: implications in clinical practice. Current cardiology reviews. 2013 Aug 1;9(3):197-210.

2. Aro AL, Huikuri HV, Tikkanen JT, Junttila MJ, Rissanen HA, Reunanen A, Anttonen O. QRS-T angle as a predictor of sudden cardiac death in a middle-aged general population. Europace. 2012 Jun 1;14(6):872-6.

3. Kardys I, Kors JA, van der Meer IM, Hofman A, van der Kuip DA, Witteman JC. Spatial QRS-T angle predicts cardiac death in a general population. European heart journal. 2003 Jul 1;24(14):135764.

4. Oehler A, Feldman T, Henrikson CA, Tereshchenko LG. QRS-T angle: a review. Annals of Noninvasive Electrocardiology. 2014 Nov;19(6):534-42.

5. Cortez D, Sharma N, Devers C, Devers E, Schlegel TT. Visual transform applications for estimating the spatial QRS-T angle from the conventional 12-lead ECG: Kors is still most Frank. Journal of electrocardiology. 2014 Jan 1;47(1):12-9.

6. Cortez DL, Schlegel TT. When deriving the Spatial QRS-T angle from the 12-lead electrocardiogram, which transform is more Frank: regression or inverse Dower?. Journal of Electrocardiology. 2010 Jul 1;43(4):302-9.

7. ter Haar CC, Maan AC, Warren SG, Ringborn M, Horáček BM, Schalij MJ, Swenne CA. Difference vectors to describe dynamics of the ST segment and the ventricular gradient in acute ischemia. Journal of electrocardiology. 2013 Jul 1;46(4):302-11.

8. Li YH, Ren XJ, Han ZH, Wang YL, Wang Y, Zhang JR, Chen F. Value of the frontal planar QRS-T angle on cardiac dysfunction in patients with old myocardial infarction. International journal of clinical and experimental medicine. 2013;6(8):688.

9. Brown RA, Schlegel TT. Diagnostic utility of the spatial versus individual planar QRS-T angles in cardiac disease detection. Journal of Electrocardiology. 2011 Jul 1;44(4):404-9.

10. Zhang X, Zhu Q, Zhu L, Jiang H, Xie J, Huang W, Xu B. Spatial/frontal QRS-T angle predicts all-cause mortality and cardiac mortality: a metaanalysis. PLoS One. 2015 Aug 18;10(8):e0136174.

11. Wellens HJ, Schwartz PJ, Lindemans FW, Buxton AE, Goldberger JJ, Hohnloser SH, Huikuri HV, Kääb S, La Rovere MT, Malik M, Myerburg RJ. Risk stratification for sudden cardiac death: current status and challenges for the future. European heart journal. 2014 Jul 1;35(25):1642-51.

12. Zapolski T, Jaroszyński A, Drelich-Zbroja A, Wysocka A, Furmaga J, Wysokiński A, Książek A, Szczerbo-Trojanowska M, Rudzki S. Aortic stiffness, left ventricle hypertrophy, and homogeneity of ventricle repolarization in adult dialyzed patients. The Scientific World Journal. 2012 Jan 1;2012.

13. Voulgari C, Moyssakis I, Perrea D, Kyriaki D, Katsilambros N, Tentolouris N. The association between the spatial QRS-T angle with cardiac autonomic neuropathy in subjects with Type 2 diabetes mellitus. Diabetic medicine. 2010 Dec;27(12):1420-9.

14. Akar FG, Rosenbaum DS. Transmural electrophysiological heterogeneities underlying arrhythmogenesis in heart failure. Circulation research. 2003 Oct 3;93(7):638-45.

15. Borleffs CJ, Scherptong RW, Man SC, van Welsenes GH, Bax JJ, van Erven L, Swenne CA, Schalij MJ. Predicting ventricular arrhythmias in patients with ischemic heart disease: clinical application of the ECG-derived QRS-T angle. Circulation: Arrhythmia and Electrophysiology. 2009 Oct $1 ; 2(5): 548-54$. 